\title{
Automatic Image Registration through Histogram Based Image Segmentation
}

\author{
${ }^{1}$ Mr. Selva Raj K, ${ }^{2}$ Mr.R. Poovendran M.E., \\ ${ }^{I}$ M.E in Communication Systems (ECE), Final year Adhiyamaan College of Engineering, Hosur, India \\ ${ }^{2}$ Asst Prof, ECE Dept., Adhiyamaan College of Engineering, Hosur, India
}

\begin{abstract}
Automatic image registration is still an actual chal- lenge in several fields. Although several methods for automatic image registration have been proposed in the last few years, it is still far from a broad use in several applications, such as in remote sensing. In this paper, a method for automatic image registration through histogram-based image segmentation (HAIRIS) is proposed. This new approach mainly consists in combining several segmentations of the pair of images to be registered, according to a relaxation parameter on the histogram modes de- lineation (which itself is a new approach), followed by a consistent characterization of the extracted objects-through the objects area, ratio between the axis of the adjust ellipse, perimeter and fractal dimension - and a robust statistical based procedure for objects matching. The application of the proposed methodology is illustrated to simulated rotation and translation. The first dataset consists in a photograph and a rotated and shifted version of the same photograph, with different levels of added noise. It was also applied to a pair of satellite images with different spectral content and simulated translation, and to real remote sensing examples comprising different viewing angles, different acquisition dates and different sensors. An accuracy below 1 for rotation and at the subpixel level for translation were obtained, for the most part of the considered situations. HAIRIS allows for the registration of pairs of images (multitemporal and multisensor) with differences in rotation and translation, with small differences in the spectral content, leading to a subpixel accuracy.
\end{abstract}

Index Terms: Histogram, image registration, image segmenta- tion, matching, Wiener filtering.

\section{Introduction}

AUTOMATIC image registration (AIR) is still a present challenge regarding image processing related applications. Reviews concerning image registration methods can be

found in [3] and [45]. Remote sensing applications is one of the fields where further research on AIR methods is required.

Manuscript received April 30, 2010; revised August 25, 2010; accepted Au- gust 26, 2010. Date of publication September 13, 2010; date of current version February 18, 2011. The work of H. Gonçalves was supported by Fundação para a Ciência e a Tecnologia, Portugal. The associate editor coordinating the review of this manuscript and approving it for publication was Dr. Brian D. Rigling.

H. Gonçalves is with the Departamento de Geociências, Ambiente e Ordena- mento do Território, Faculdade de Ciências, Universidade do Porto, 4169-007

Porto, Portugal. He is also with the Centro de Investigação em Ciências Geo-Es-paciais, Universidade do Porto (e-mail: hernani.goncalves@fc.up.pt).

J. A. Gonçalves is with the Departamento de Geociências, Ambiente e Orde- namento do Território, Faculdade de Ciências, Universidade do Porto, 4169-007

Porto, Portugal (e-mail: jagoncal@fc.up.pt).

L. Corte-Real is with the Departamento de Engenharia Electrotécnica e de Computadores, Faculdade de Engenharia, Universidade do Porto and with

Under this scope, there are particular difficulties so that AIR methods suitable for many computer vision applications will present limited performance.

The rigid-body model under the scope of automatic image registration methods is still a present subject of research [22], in particular under the scope of remote sensing applications [2], [40]. The problem of registering remote sensing images can roughly be locally seen as the determination of translations and a small rotation. Under the scope of computer vision applications, the rigid-body transformation may seem a simple problem to solve with many existing methods. However, under the scope of remote sensing applications, one of the major problems is related to the radiometric content (due to multisensor or multi- spectral pairs of images). Moreover, scale is frequently known, as most satellite images are provided with sufficiently accurate scale information, being the exception aerial photographs when the aircraft is flying across a region with significant differences in the terrain elevation. Similar comments may be applicable regarding differences in the view-angle, as satellite images are roughly acquired at an altitude of $600 \mathrm{~km}$, being the affine or homography mostly required for 
aerial photographs under the circumstances previously mentioned for aerial photographs.

Over the last 30 years, a large amount of articles has been pub- lished regarding the topic image segmentation, either related to methods themselves or their application to several fields such as medicine, remote sensing, among others [10], [32], [33], [38], [43]. However, image segmentation (IS) is still an actual field of research, regarding automatic methods of image processing. IS is generally defined as the process that partitions an image into regions, each of them fulfilling a given criteria, which can be from the image domain and/or feature space. From image seg- mentation methods, we expect the extraction of a set of objects present on an image, as we visually detect them. In other words, it is expected that a segmentation method acts as artifical intelligence on the identification of objects on a scene. However, the objective of the segmentation may be quite subjective, de- pending upon the detail and features we are expecting. For in- stance, on the segmentation of an image of a human body, one may be interested in delineating the whole body as a single ob- ject, or its constituent parts, which may become itself quite sub- jective. Regarding remote sensing applications, this aspect may become even more complex [28].

Image segmentation comprises a wide variety of methods [8], [33], either for monochrome or color images (or to a single

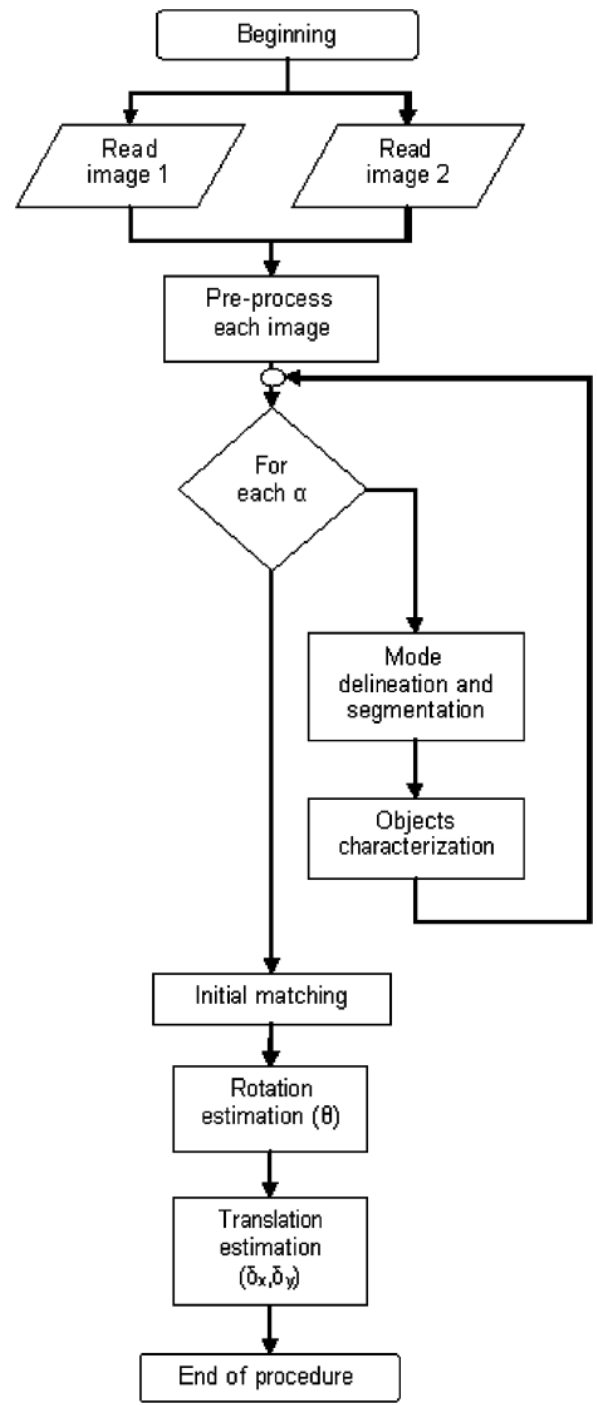

Fig. 1. Flowchart of the main steps of HAIRIS.

Enhancement step prior further processing. By image enhance- ment (which is itself a largely subjective process), it is intended to obtain an image with less detail than the original version, nearest to the "object" identification which is performed by the human eye. Although typically more mathematical and com- plex, restoration algorithms may provide the exploitation of the detailed characteristics of an image and its degradation [25]. Despite the main purpose of image restoration methods is to model and remove the degradation, these methods may also be used with other purposes. Since with image segmentation it is intended to extract objects 
(in particular their boundaries), one may view the image objects which have some texture as a kind of degradation. Therefore, it is intended to remove that degra- dation, which is assumed to be additive random noise.

The Wiener filter [25] is one of the most used filters under the scope of image restoration methods [17]. However, it may also be used for image enhancement, with the aim of reducing the detail on an image, since it is typically a lowpass filter and consequently induces a significant blurring effect. Its frequency response $H\left(\omega_{1}, \omega_{2}\right)$ is given by

$H\left(\omega_{1}, \omega_{2}\right)$

$$
=\frac{P_{f}\left(\omega_{1}, \omega_{2}\right)}{P_{f}\left(\omega_{1}, \omega_{2}\right)+P_{v}\left(\omega_{1}, \omega_{2}\right)}
$$

where $\quad$ stands for the original image power spectra an $\left(\omega_{1}, \omega_{2}\right) \quad$ for the additive random noise power spectra. Although one might be interested in inducting $\xi_{\omega}$ sme) blur- ring on the image, the use of a fixed (space-invariant) filter throughout the image may decrease the clearness of the ob- jects boundaries. Therefore, an adaptive image restoration is required. A "pixel-by-pixel processing" approach may become quite computationally expensive, in opposition to a "subimage-by-subimage processing" where we divide the image into a certain number of tiles. The latter is typically considered for subimages with size between 8 8 and 32

32 pixels [25]. However, since the objective of the Wiener filter employment is different from restoration, it is advisable to con- sider the conservative smallest possible square tile (beyond a single pixel) size of 33 pixels. Although this latter approach might induce the so-called "blocking effect," it may be ignored for images with low SNR. $x$

Recalling the additive random noise, we generally have no a priori information, which may be overcome by considering measured features such as the local variance, providing the de- termination of the presence of significant high-frequency de- tails. Since we are interested in delineating the objects bound- aries, an edgesensitive adaptive image restoration version of the Wiener filter [25] is adequate. This method is based upon the idea of reducing more noise near edges without additional edge blurring through a cascade of 1-D adaptive filters. Let $\leq \leq N$ ) represent a 1-D (space variant) filter, where

is four and represents the four directions corresponding to the

angles of $0,45,90$, and 135 . Then, these operators are se- quentally applied, which lead to an improvement over the per- formance of some 2-D adaptive restoration algorithms, and to typically less computational requirements [25].

Additionally, in order to overcome significant differences $[\cdot b e-$ tween the histograms of the images to be registered, an his- togram equalization of image 2 using the histogram counts of image 1 is performed, prior to the application of the Wiener filter. In this way, the Wiener filtering on image 2 allows both for the reduction of the image detail, as well as to the smoothing of the histogram, which becomes spiky due to the histogram equalization step.

\section{B. Histogram-Based Segmentation: Mode Delineation and Image Segmentation}

The method used for mode delineation is based upon the anal- ysis of the consecutive slopes of the histogram. Let $x(m)$ be the image histogram counts, and

$$
\begin{gathered}
\multicolumn{3}{c}{m=0, \ldots, M} \\
y(n) \quad x(n \quad 1), \quad 1, \ldots, M
\end{gathered}
$$




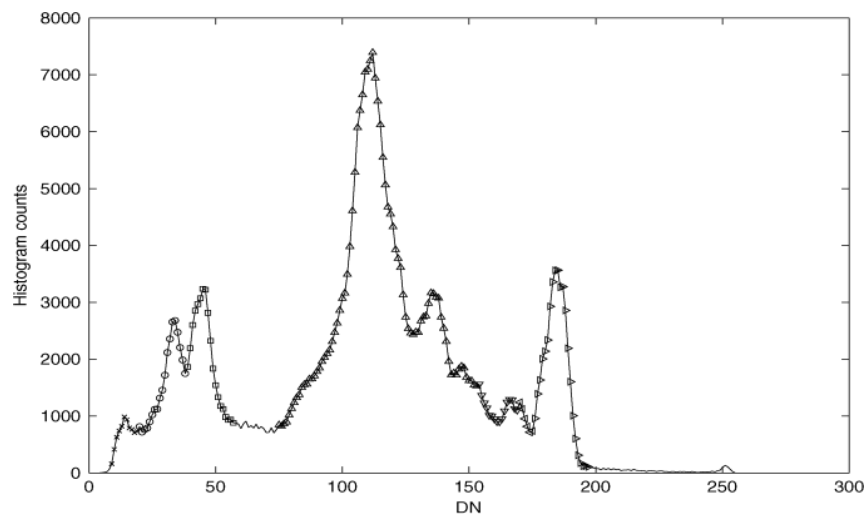

(a)
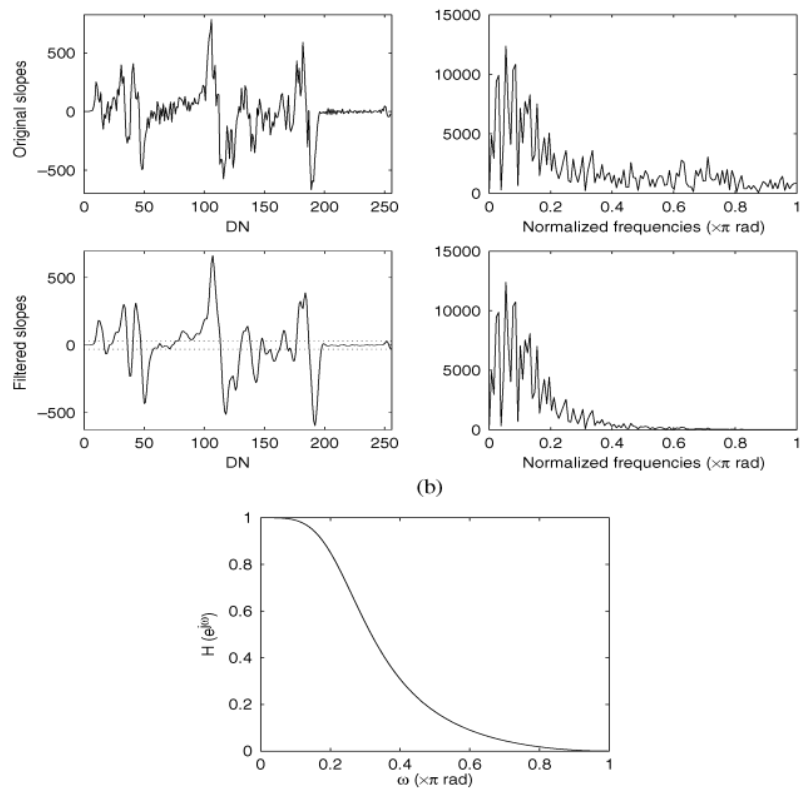

Fig. 2. (a) Histogram of the image presented in Fig. 4(a), where the different superimposed symbols represent the seven identified Modes $25(+)$ Slopes sequence (on the left) corresponding to the histogram in (a), before (upper plots) and after (lower plots) the application of the low-pass filter represented in (c), and their representation in the frequency domain (on the right). The limits (horizontal dashed lines) superimposed on the filtered slopes sequence correspond to the $99 \%$ confidence interval (further details in the text). (c) Frequency response of the low-pass filter applied to the original slopes sequence in (b). the sequence of the consecutive slopes, where is the number of histogram levels ( for an 8-b image). The idea behind this approach is to choose an adequate threshold for considering whether or not one is in the presence of a mode, which is characterized by a significant increase and/or decrease on the slopes sequence. As illustrated by the example in Fig. 2, a mode is characterized by extreme positive and negative values of the slopes sequence. Therefore, one obvious and functional solution for delineating a mode is to obtain a confidence interval for the slopes sequence, where the presence of a mode is de- tected by the slopes which are outside the $99 \%$ confidence in- terval [Fig. 2(b)]. The t-Student based confidence interval for the mean is adequate for the most common 8-b images, since it corresponds to 256 points which is sufficiently higher than 


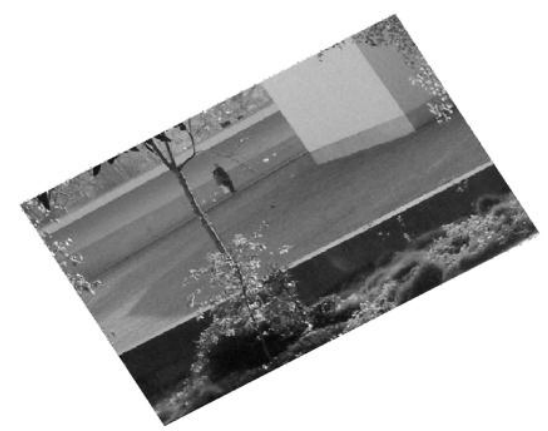

(a)

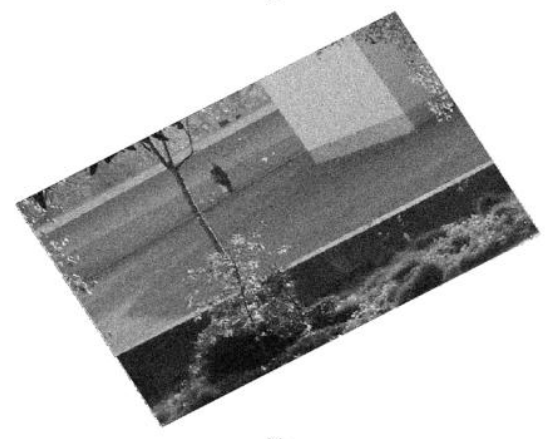

(c)

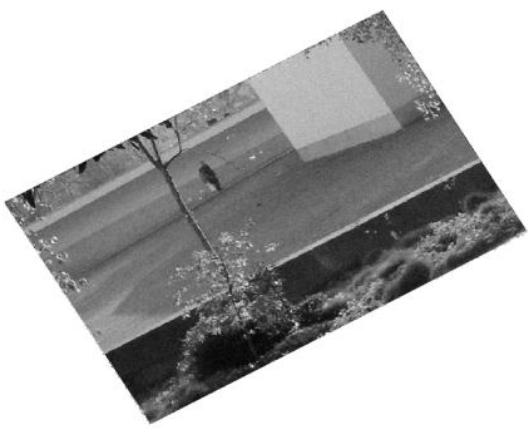

(b)

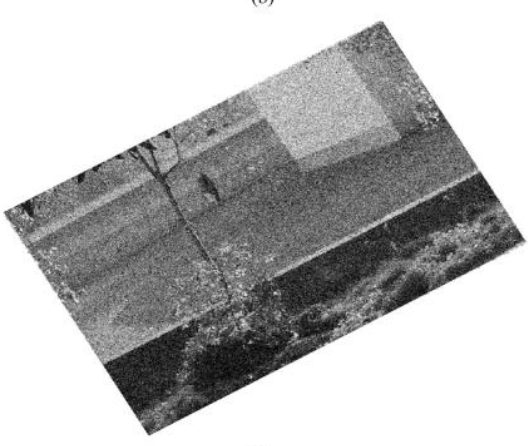

(d)

Fig. 5. Image represented in Fig. 4(b), after the addition of white Gaussian noise with equal to (a) 10\%, (b) $20 \%$, (c) $50 \%$, and (d) $100 \%$ of the image standard deviation.

A panchromatic image from the same region, acquired by the sensor IKONOS (Fig. 9), with an initial approximate geometric correction part of the product (with a spatial resolution of $1 \mathrm{~m}$ ). The temporal difference between these-images is around one year, where significant changes such as new buildings (left part of the image) can be observed during this period. Additionally, beyond the difference between the sensors, it can be clearly seen the effect of different viewing angles (IKONOS image was ac- quired at a viewing angle of around 20 ), which is associated to significant shadow effects (Fig. 9). All these differences in this pair of images makes this example a quite difficult pair to register (Fig. 9). The displacement between these two im- ages was manually obtained, through a difficult identification of six conjugate points. The horizontal shifts between these con- jugate points ranged between 16.25 and 23.25 (average of 20.75 and standard deviation of 2.77), whereas the vertical shifts ranged between 2.25 and 4.25 (average of 3.38 and standard deviation of 0.70). These differences are explained by the set of significant differences previously mentioned. Through the least squares method a null rotation was found between this pair of images and considering the six identified conjungate points. Also in this example, HAIRIS led to acceptable results considering the average and standard deviation of the reference values, despite all the difficulties contained in this pair of im- ages (Table I).

\section{Comparison of HAIRIS With Other Methods}

In order to establish a bāseline for comparison of HAIRIS with other AIR methods, two popular methods of AIR have been considered: scale invariant feature transform (SIFT) [26] and a contour-based approach [14], [23]. Since the former method (implementation of the author) provides a set of con- jugate points, the parameters,$\delta_{x}$ and $\delta_{y}$ were estimated through the least squares method [29] (once redundancy is guaranteed) and according to (1). We considered the distance ratio equal to 0.6 , as the value 0.8 suggested in [26] led to worst results in both photographs and remote sensing images. The implementation of the later method allows for considering the RST transformation (and not merely RT), and the default parameters have been considered. The results obtained from applying these two methods to the previously considered pairs of images are presented in Table II, including the associated computational time.

It can be observed that HAIRIS generally outperformed SIFT and the countour-based approach, in particular for the remote sensing examples. Although SIFT is a powerful method under the scope of computer vision applications, its performance is quite limited for remote sensing images [30], [39]. Furthermore, its dependence upon the parameter associated to the ratio of distances is an undesirable property under the scope of fully AIR methods [26]. The contour-based approach [14], [23] ex- hibited in general the worst performance. Although it is a quite fast method, it is not able to lead with more complex situations such as significant presence of noise or multisensor pairs of im- ages (Table II), whereas HAIRIS had accurately registered these complex pairs of images (Table I). Although higher computa- tional times were associated with HAIRIS (mainly related to the segmentation stage), it is worth to mention that we implemented HAIRIS in MATLAB (the contour-based 
approach implemen- tation is based upon $\mathrm{C}++$ which is faster) and the code is far from being optimized, since several outputs are produced given the experimental state of the method. Moreover, it showed to be able to register pairs of images covering a wide range of situa- tions (multitemporal, multisensor, and in the presence of noise).

\section{Discussion and Conclusion}

A large variety of automatic image registration methods can be found in [3] and [45]. In several applications, the registration model only assumes rotation and translation [22], where the reg- istration of satellite images is an example [2], [40]. In this paper, a new approach for automatic image registration through histogram-based image segmentation (HAIRIS) is proposed, with

clear advantages by joining these two main areas of image pro- cessing. Since HAIRIS does not require any search interval ei- ther for rotation or translation, it is a fully automatic procedure. With the filtering step - an important preprocessing stage of the proposed methodology - the objective is to transform the original image in order to take advantage of the psychophys- ical aspects of the human visual system. The Wiener filter is one of the solutions among several other possible alternatives. The proposed Wiener filtering, although with its associated dis- advantages, showed to be adequate for the variety of examples considered in this manuscript.

\section{References}

[1] A. Z. Arifin and A. Asano, "Image segmentation by histogram thresh- olding using hierarchical cluster analysis," Pattern Recognit. Lett., vol. 27, pp. 1515-1521, 2006.

[2] Y. Bentoutou, N. Taleb, K. Kpalma, and J. Ronsin, "An automatic image registration for applications in remote sensing," IEEE Trans. Geosci. Remote Sens., vol. 43, no. 9, pp. 2127-2137, Sep. 2005.

[3] L. G. Brown, "A survey of image registration techniques," Comput. Surv., vol. 24, no. 4, pp. 325-376, 1992

[4] J. H. Chang, K. C. Fan, and Y. L. Chang, "Multi-modal gray-level his- togram modelling and decomposition," Image Vis. Comput., vol. 20, pp. 203-216, 2002.

[5] C. I. Chang, Y. Du, J. Wang, S. M. Guo, and P. D. Thouin, "Survey and comparative analysis of entropy and relative entropy thresholding techniques,” IEE Proc.-Vis. Image Signal Process., vol. 153, no. 6, pp. 837-850, 2006.

[6] H. Chen, P. K. Varshney, and M. K. Arora, "Performance of mutual in- formation similarity measure for registration of multitemporal remote sensing images," IEEE Trans. Geosci. Remote Sens., vol. 41, no. 11, pp. 2445-2454, Nov. 2003.

[7] H. D. Cheng and Y. Sun, “A hierarchical approach to color image seg- mentation using homogeneity,” IEEE Trans. Image Process., vol. 9, no. 12, pp. 2071-2082, Dec. 2000.

[8] H. D. Cheng, X. H. Jiang, Y. Sun, and J. Wang, “Color image seg- mentation: Advances and prospects,” Pattern Recognit., vol. 34, pp. 2259-2281, 2001.

[9] M. Cheriet, J. N. Said, and C. Y. Suen, “A recursive thresholding tech- nique for image segmentation,” IEEE Trans. Image Process., vol. 7, no. 6, pp. 918-921, Jun. 1998.

[10] G. B. Coleman and H. C. Andrews, "Image segmentation by clus- tering,” Proc. IEEE, vol. 67, no. 5, pp. 773-785, May 1979.

[11] T. M. Cover and J. A. Thomas, Elements of Information Theory. Hoboken, NJ: Wiley, 1991.

[12] P. Dare and I. Dowman, "An improved model for automatic feature- based registration of SAR and SPOT images," J. Photogram. Remote Sens., vol. 56, pp. 13-28, 2001.

[13] E. Dudewicz and S. Mishra, Modern Mathematical Statis- tics. Hoboken: Wiley, 1988.

[14] D. Fedorov, L. M. G. Fonseca, C. Kenney, and B. S. Manjunath, "Au- tomatic registration and mosaicking system for remotely sensed im- agery," in Proc. Image Signal Process. Remote Sens. VIII, 2003, vol. 4885, pp. 444-451.

[15] M. Frigge, D. C. Hoaglin, and B. Iglewicz, "Some implementations of the boxplot," Amer. Statist., vol. 43, no. 1, pp. 50-54, 1989.

[16] H. Gonçalves, J. A. Gonçalves, and L. Corte-Real, "Measures for an objective evaluation of the geometric correction process quality,” IEEE Geosci. Remote Sens. Lett., vol. 6, no. 2, pp. 292-296, Apr. 2009.

[17] R. C. Gonzalez and R. E. Woods, Digital Image Processing. Upper Saddle River, NJ: Prentice-Hall, 2002.

[18] A. Goshtasby, G. C. Stockman, and C. V. Page, "A region-based approach to digital image registration with subpixel accuracy," IEEE Trans. Geosci. Remote Sens., vol. GE-24, no. 3, pp. 390-399, May 1986.

[19] B. K. P. Horn, "Closed-form solution of absolute orientation using unit quaternions,” J. Opt. Soc. Amer. A, Opt. Image Sci., vol. 4, no. 4, pp.629-642, 1987.

[20] J. Inglada and A. Giros, “On the possibility of automatic multisensor image registration,” IEEE Trans. Geosci. Remote Sens., vol. 42, no. 10, pp. 2104-2120, Oct. 2004.

[21] J. N. Kapur, P. K. Sahoo, and A. K. C. Wong, "A new method for gray-level picture thresholding using the entropy of the histogram," Comput. Vis. Graph. Image Process., vol. 29, no. 3, pp. 273-285, 1985 .

[22] G. Lazaridis and M. Petrou, "Image registration using the walsh trans- form,” IEEE Trans. Image Process., vol. 15, no. 8, pp. 23432357, Aug. 2005

[23] H. Li, B. S. Manjunath, and S. K. Mitra, “A countour-based approach to multisensor image registration,” IEEE Trans. Image Process., vol. 4, no. 3, pp. 320-334, Mar. 1995.

[24] Z. P. Liang, H. Pan, R. L. Magin, N. Ahuja, and T. S. Huang, "Au- tomated image registration by maximization of a region similarity 